\title{
Synaptic Learning of the Resonator Network Interacting with Oscillatory Background and Noise
}

\author{
Taishi Matsumura, Tatsuo Kitajima, Tetsuya Yuasa, Siu Kang* \\ Department of Bio-Systems Engineering, Graduate School of Science and Engineering, Yamagata University, \\ Yonezawa, Yamagata, Japan; *siu@yz.yamagata-u.ac.jp
}

SNE 28(3), 2018, 125 - 127, DOI: 10.11128/sne.28.sn.10434 Received: Sept. 15, 2016 (Selected EUROSIM Congress 2016 Postconf. Publ.), Revised July 30, Accepted: August 25, 2018 SNE - Simulation Notes Europe, ARGESIM Publisher Vienna, ISSN Print 2305-9974, Online 2306-0271, www.sne-journal.org

Abstract. Rhythmic activities were widely observed in many brain regions. Human EEG recording revealed several frequency modulation of the oscillation reflecting internal brain states such as attentional modulation in visual systems. On the other hand, in vivo intracellular recordings suggested that individual neurons showed persistent membrane fluctuations and global oscillation originated from the activity of the neuronal fluctuations. Furthermore, it was found that some types of neuron showed membrane resonance in their subthreshold level. However, functional roles of the subthreshold resonance in a recurrent neural network are still unknown. Here, we computationally examined the behavior of resonator network driven by external inputs and organized through the spike-timing-dependent plasticity (STDP) under oscillatory background and noise. As a result, it was shown how the resonator network modified its responsiveness depending on frequency modulation and its connectivity through the STDP.

\section{Introduction}

Oscillatory activities in brain often reflect internal states. For example, attentional level in visual systems is characterized by the intensity of gamma frequency component in oscillation. In a sleep stage classification, rapid-eye-movement (REM) sleep and non-REM sleep are typically defined by theta and delta powers in EEG.

On the other hands, several intracellular recordings demonstrated membrane potential fluctuations in individual neurons. Importantly, theoretical and experi- mental studies revealed that the fluctuation did not mean a disadvantage but lead to high advantage of neural computation.

This work was supported by JSPS KAKENHI Grant Number 16K00386.

In addition, it is well known that synaptic plasticity is a key factor of brain computation such as learning and memory. Recent experimental study reported the STDP that potentiates transmission efficacy if pre-synaptic neuron elicits a spike before post-synaptic neuron. On the other hand, the STDP depresses it when post-firing proceeds pre-firing [1]. The STDP is considered as an important neural basis of transformation between spatial- and temporal-information because the plasticity includes relative timings of spikes. In addition, theoretical studies suggest that the STDP has computational advantage [2].

Furthermore, recent intracellular recording study reported that some types of neurons in hippocampus and entorhinal cortex showed a relatively strong response to specific frequency when they receive sinusoidal current injection whose frequency changes in time-dependent manner such as a chirp current. The response property of a resonator neuron in isolated condition was well examined and ionic mechanism on the generation of resonance was identified. However, how the resonators behave in recurrent networks, especially with synaptic learning, is still unclear.

Here, we performed numerical simulation of the network with subthreshold resonance. In our previous study, the frequency selectivity of network connectivity and automatic tuning for optimal noise intensity were already demonstrated under the fixed timing of external inputs [3]. In the present study, we examined how much the network organization was robust for the external stimuli with various temporal jitters and randomness. 


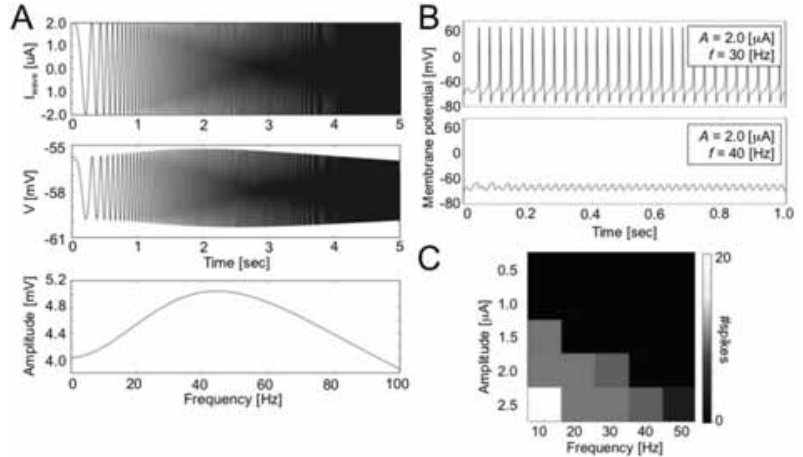

Figure 1. Neuronal response to oscillatory inputs.

\section{Method}

\subsection{Neuron model}

We employed the resonator neuron model described by Acker, et al. [4]. The resonator neuron exhibits subthreshold resonance under isolated conditions due to its intrinsic properties such as persistent sodium, slow and non-inactivating potassium, hyperpolarization-activated cation channels. Dynamics of the membrane potential is described with the Hodgkin-Huxley formalism.

\subsection{Network organization}

Our network included 100 excitatory neurons. All the neurons had excitatory synapses mediated by first order kinetics and all-to-all connectivity in the initial state. Self-excitation was prohibited and all synaptic weights were modified through the STDP. The STDP potentiated or depressed individual synapses depending on the temporal order between pre- and post-synaptic firings.

\section{$1.3 \quad$ Input currents}

Our model network receives four types of input currents. All neurons in the network receive a bias current, $I_{a p p}$, in order to generate subthreshold resonance the same as the original model. Background oscillatory activity, $I_{\text {wave }}$, is described as sinusoidal current injection whose frequency is modulated, corresponding to the internal state of a brain. Background noise, $I_{\text {noise }}$, is given as the Gaussian white noise with a diffusion constant $D$. External stimulations, $I_{\text {stim }}$, are applied onto 20 neurons, and their timing is 2 millisecond-earlier than every peaks of the $I_{\text {wave }}$. In some simulations, we introduced temporal jitters to these stimuli in the time-fixed or stochastic manner.
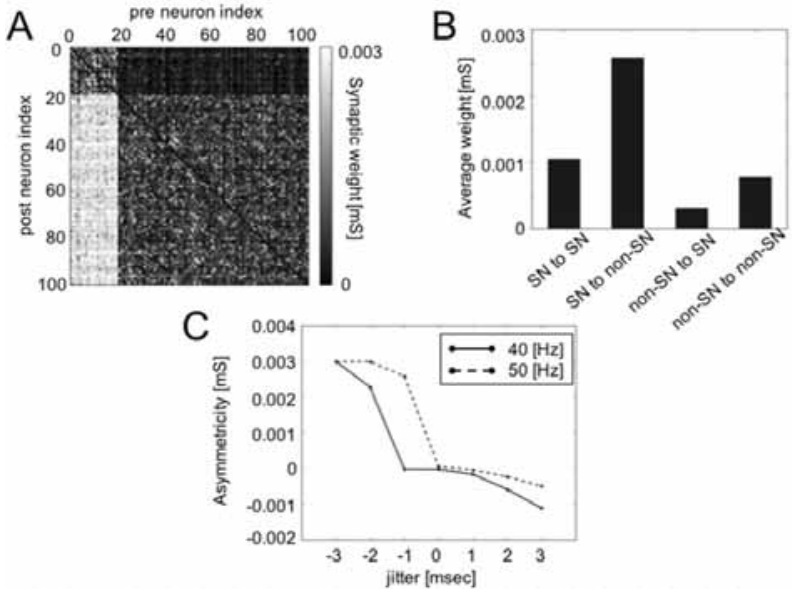

Figure 2. Synaptic weights after the STDP learning.

\section{Result}

\subsection{Responsiveness of single neuron to oscillatory inputs}

Neuronal responses to oscillatory inputs with various frequencies and amplitudes are shown in Figure 1. Each panel indicates an input current (upper), membrane potential as output (middle), and response amplitude (lower) (Figure 1A). The response to different frequencies is indicated in Figure 1B. Upper and lower panel respectively shows the time course of the membrane potential driven by $30 \mathrm{~Hz}$ and $40 \mathrm{~Hz}$ with the same amplitude. The neuron showed supra-threshold response, that is, spike firings in some parameter ranges. Figure 1C shows spike outputs for various parameters of oscillation. The heat map indicates the number of spikes. The $\mathrm{x}$-axis is frequency and the $\mathrm{y}$-axis the amplitude of the oscillatory current. Difference in responsiveness is most prominent in the modulation between $40 \mathrm{~Hz}$ or $50 \mathrm{~Hz}$ of oscillation.

\subsection{Synaptic learning through the STDP}

Figure 2 indicates the synaptic learning of the network driven by the external inputs under oscillation and noise. Through the STDP learning, the model network is gradually modified such that the synapses from the stimulated neurons (SN) to the non-stimulated neurons (non-SN) are potentiated. On the other hand, synapses from non-SN to $\mathrm{SN}$ are depressed (Figure 2A, B; $A=2 \mu \mathrm{A}, f=40 \mathrm{~Hz}$, jitter $=-2 \mathrm{msec})$. Furthermore, such an asymmetric connectivity was alternated through the frequency modulation of oscillatory background. 
The model network showed the automatic tuning of frequency selectivity for noise intensity such that 1) asymmetric connectivity or symmetric connectivity was organized frequency-independently under extremely weak or strong noise, respectively, 2) asymmetric and symmetric connectivity was frequency-dependently organized under the moderate noise intensity. Such the frequency selectivity robustly appeared even with the temporal jitter of external stimuli that proceeded to peaks of oscillation. On the other hand, if the timing of external stimuli had delay from the peaks, the frequency selectivity disappeared (Figure 2C).

\section{Disucussion}

The present computational study demonstrates several properties of a resonator network. Through the formation of non-uniform connectivity, the resonator network could store the spatial information of external stimuli in frequency-dependent manner. Furthermore, such the selectivity of frequency was automatically tuned for moderate noise even under the redundancy of external stimuli such as temporal jitter. It is possible to expect that frequency-dependent neural processing such as attentional modulation in visual systems had a neural basis shown in present study.

\section{Acknowledgement}

The authors thank Masanari Minamisawa, Kana Kimura for fruitful discussion on the research.

\section{References}

[1] Bi GQ, Poo MM. "Synaptic modifications in cultured hippocampal neurons: dependence on spike timing, synaptic strength, and postsynaptica cell type.”, The Journal of Neuroscence., 1998, Dec 15;18(24):10464-72.

[2] Song s, Miller KD, Abbott LF. "Competitive Hebbian learning through Spike-timing-dependent synaptic plasticity," Nature Neuroscience, Sep:3(9), 2000, 916-926.

[3] Kang S, Minamisawa M, Kimura K, Matsumura T, Kitajima T, Yuasa T. "The learning property of spiketiming-dependent plasticity in neural network with the subthreshold membrane resonance", Organized Session 15 "Complex Networks and Complex Systems" on International Conference on Simulation Technology, JSST2015.

[4] Acker CD, Kopell N, White JA: "Synchronization of Stronglly Coupled Excitatory Neurons: Relating Network Behavior to Biophysics", The Journal of Computational Neuroscience, 2001, 15:71-90. 